\title{
SÍNTESE, CARACTERIZAÇÃO E ESTUDO DAS PROPRIEDADES DE UM NOVO COMPLEXO MONONUCLEAR CONTENDO QUERCETINA E ÍON Ga(III)
}

Vanessa do Nascimento Simões, Lis Regiane Vizolli Favarin, Natália Aparecida Cabeza, Tamires Donizeth de Oliveira, Antonio Rogério Fiorucci, Jusinei Meireles Stropa, Daniela Cristina Manfroi Rodrigues, Alberto Adriano Cavalheiro e Ademir dos Anjos*

Universidade Estadual de Mato Grosso do Sul, Unidade Universitária de Naviraí, 79950-000 Naviraí - MS, Brasil

Recebido em 27/2/12; aceito em 23/10/12; publicado na web em 20/2/13

\begin{abstract}
SYNTHESIS, CHARACTERIZATION AND STUDY OF THE PROPERTIES OF A NEW MONONUCLEAR QUERCETIN COMPLEX CONTAINING Ga(III) IONS. Flavonoids are one of the most important compound groups applied as medicine given their antioxidant properties, but several intrinsic properties can be improved through structural modifications to their molecules. Here, the synthesis and characterization of a new gallium (III) complex with quercetin is described. Electrochemical properties, as well as antioxidant and cytotoxic activities, were investigated and compared to the free flavonoid molecule. The mononuclear complex obtained, $\left[\mathrm{Ga}\left(\mathrm{C}_{15} \mathrm{H}_{9} \mathrm{O}_{7}\right)_{3}\right] \cdot 2 \mathrm{H}_{2} \mathrm{O} \cdot 2 \mathrm{CH}_{3} \mathrm{OH} \cdot \mathrm{CH}_{3} \mathrm{CH}_{2} \mathrm{OH}$, seems more active as a DPPH radical scavenger given its lower oxidation potential compared to quercetin. The new complex cytotoxic responses have shown to be more effective than those of the free flavonoid and of lapachol used as a control.
\end{abstract}

Keywords: quercetin; gallium(III) complex; biological activities.

\section{INTRODUÇÃO}

Os flavonoides são compostos de baixa massa molar com uma estrutura base constituída por dois anéis aromáticos (A e B) ligados através de um anel pirano (C), como pode ser visualizado na Figura 1. Eles representam um dos grupos fenólicos mais importantes e diversificados entre os produtos de origem natural, sendo amplamente distribuídos no reino vegetal. ${ }^{1}$ Nos últimos anos essas moléculas têm atraído a atenção de muitos pesquisadores, pois exibem notável variedade de atividades biológicas, físicas e farmacológicas. ${ }^{2,3}$ Elas podem atuar como sequestradores de radicais livres, neutralizando espécies reativas de oxigênio, e como ligantes quelatos para íons metálicos, sendo que se atribui a essas características a responsabilidade por suas propriedades benéficas. ${ }^{4,5}$<smiles></smiles>

Figura 1. Representação esquemática da estrutura do flavonoide quercetina

A quercetina (Figura 1) é um dos flavonoides mais presentes na dieta humana, pois é encontrada em grande quantidade nas frutas, verduras e chás. Possui propriedades de grande interesse, entre elas estão seus efeitos anticarcinogênicos, protetores do sistema renal, cardiovascular e hepático. ${ }^{6} \mathrm{~A}$ interação dos flavonoides com íons metálicos pode mudar as propriedades antioxidantes e alguns outros efeitos biológicos, sendo a maioria de suas propriedades muitas vezes superiores às dos compostos originais. ${ }^{3}$ Pesquisas têm mostrado que

*e-mail: piu_floripa@uems.br alguns complexos metálicos com a quercetina possuem resistência à atividade neoplasmática e apresentam atividades antisséptica, anti-inflamatória e/ou antioxidante. ${ }^{7}$

O gálio atua em nosso organismo em focos de inflamação e infecção particularmente nos neutrófilos granulosos e leucócitos polimorfonucleares. ${ }^{8} \mathrm{O}$ principal interesse clínico do gálio deriva da observação de que suas propriedades metabólicas são similares às do ferro. ${ }^{9-11}$ Desde os anos 70, o gálio mostrou-se eficaz na diminuição da reabsorção óssea acelerada, redução associada aos níveis plasmáticos elevados de cálcio ou inibidores da proliferação neoplásica, tornando-se o segundo íon metálico depois da platina, a ser utilizado no tratamento de câncer. ${ }^{10}$ Entretanto, seus sais trivalentes $\left(\mathrm{Ga}^{3+}\right)$ são fracamente absorvidos pelo intestino e alguns derivados são susceptíveis à hidrólise, diminuindo, assim, sua eficiência biológica. ${ }^{11}$ Por isso o grande interesse na síntese de compostos de coordenação com o íon metálico, visando uma possível correção dessas deficiências. Estudos demonstram um aumento da biodisponibilidade e uma eficácia antitumoral superior para alguns dos complexos, comparado com o nitrato de gálio(III) e outros sais do íon metálico. ${ }^{10}$ Kopacz e colaboradores ${ }^{12-14}$ descrevem a síntese, algumas propriedades físico-químicas e estudos potenciométricos de complexos formados entre a quercetina e o ácido quercetina-5-sulfônico com metais do grupo 13 (alumínio, gálio e indio), entretanto, não fornecem uma caracterização estrutural efetiva, bem como estudos relacionados às atividades biológicas desses compostos.

Desta forma, o presente trabalho descreve a síntese, caracterização e estudo das propriedades eletroquímicas e biológicas (antioxidante e citotóxica) de um novo complexo metálico obtido a partir da reação entre o flavonoide natural quercetina e íon $\mathrm{Ga}^{3+}$.

\section{PARTE EXPERIMENTAL}

\section{Materiais e métodos}

Todos os reagentes e solventes empregados nas sínteses e análises foram adquiridos de fontes comerciais e utilizados sem purificação prévia. 
Análise elementar, espectroscopia (IV e UV-Vis) e estudo térmico

A determinação dos percentuais de carbono e hidrogênio foi realizada em analisador elementar de CHNS Thermo Cientific Flash 2000. A análise termogravimétrica do complexo foi obtida em um analisador térmico TGA Q50 da TA Instrument, em uma faixa de temperatura de 25 a $550{ }^{\circ} \mathrm{C}$, com gradiente de aquecimento de 20 ${ }^{\circ} \mathrm{C} \mathrm{min}{ }^{-1}$, em atmosfera de ar sintético (massa inicial da amostra: 7,5 $\mathrm{mg}$ ). As curvas da calorimetria diferencial de varredura (DSC) foram obtidas em um calorímetro TA Instruments modelo DSC Q20, em atmosferas de nitrogênio e oxigênio (ambos com vazão de $50,0 \mathrm{~mL}$ $\min ^{-1}$ ), gradiente de aquecimento de $10^{\circ} \mathrm{C} \min ^{-1}$ e faixa de varredura entre 25 a $550^{\circ} \mathrm{C}$. Os espectros na região do infravermelho (IV) foram obtidos em um espectrofotômetro Thermo Nicolet Nexus 650, com acessório de detecção por fotoacústica (resolução $8 \mathrm{~cm}^{-1}$, média de 256 espectros). Os espectros eletrônicos nas regiões do ultravioleta e visível (UV-Vis) foram obtidos em um espectrofotômetro Varian modelo Cary50, sendo as análises realizadas em metanol (grau espectroscópico) e cubetas de quartzo com capacidade para 4,0 mL com caminho óptico de $1 \mathrm{~cm}$.

\section{Ensaio citotóxico}

O ensaio de toxicidade sobre a Artemia salina foi conduzido segundo metodologia descrita na literatura ${ }^{15-17}$ com modificações. Uma solução marinha foi preparada utilizando-se sal e água destilada (38 g de sal/L de água), sendo esta dividida em duas partes, uma para a eclosão dos ovos e a outra para a preparação das diluições das substâncias-teste, com pH variando entre 8-9. Em $500 \mathrm{~mL}$ da solução salina foram adicionados aproximadamente $1,5 \mathrm{~g}$ de ovos comerciais de A. salina, submetidos a um choque de luz com lâmpada de $100 \mathrm{~W}$, por $48 \mathrm{~h}$, sendo o sistema protegido de poeira e insetos. $\mathrm{O}$ restante da solução salina foi acondicionado em outro recipiente e também submetido às mesmas condições de iluminação e temperatura. Foram utilizados $10 \mathrm{mg}$ das amostras, que foram solubilizados em metanol e o volume completado para $5 \mathrm{~mL}$ com água salinizada. Destas soluções foram retiradas alíquotas, transferidas para tubos de $5 \mathrm{~mL}$ com os volumes sendo completados com água marinha, obtendo-se concentrações de 500, 250, 100, 50 e $10 \mu \mathrm{g} \mathrm{mL}^{-1}$ para cada amostra.

Nas mesmas condições preparou-se a amostra de lapachol, que foi utilizado como controle positivo, uma vez que o composto é relatado na literatura como agente citotóxico efetivo. ${ }^{18-20} \mathrm{O}$ controle negativo apresentava solução salina e metanol. Para cada diluição foram montadas triplicatas, nas quais foram adicionadas 10 larvas de A. salina com auxílio de uma pipeta Pasteur. A contagem dos animais mortos e vivos foi realizada após $24 \mathrm{~h}$ em temperatura e iluminação ambientes com proteção contra poeira e insetos. Para obtenção dos valores de $\mathrm{DL}_{50}$ foi utilizada a análise Probit, ${ }^{21}$ através do software BioStat, ${ }^{22}$ com $95 \%$ de confiança.

\section{Avaliação da atividade antioxidante}

O ensaio antioxidante quantitativo foi realizado de acordo com modificações dos métodos descritos na literatura, ${ }^{23,24}$ monitorando-se o consumo do radical livre DPPH (2,2-difenil-1-picril-hidrazil) pelas amostras, através da medida do decréscimo da absorvância $(515 \mathrm{~nm})$ de soluções metanólicas em diferentes concentrações. Utilizou-se um espectrofotômetro Varian modelo Cary 50 e cubetas de quartzo com capacidade para 4,0 mL com caminho óptico de $1 \mathrm{~cm}$.

Primeiramente, prepararam-se $50 \mathrm{~mL}$ de uma solução de DPPH em metanol na concentração de $100 \mu \mathrm{g} \mathrm{mL}^{-1}$, mantida sob refrigeração e protegida da luz. Foram feitas diluições em triplicata de 40, 30, 20, 10,5 e $1 \mu \mathrm{g} \mathrm{mL} \mathrm{m}^{-1}$. A curva de calibração foi construída a partir dos valores da absorvância medidos a $515 \mathrm{~nm}$.

\section{Leitura das medidas de absorvâncias das amostras}

Soluções do complexo e da quercetina foram preparadas em uma concentração inicial de $100 \mu \mathrm{g} \mathrm{mL}^{-1}$ e diluídas nas seguintes concentrações: $1,5,10,15,20$ e $25 \mu \mathrm{g} \mathrm{mL} \mathrm{m}^{-1}$. As medidas das absorvâncias das misturas reacionais $(0,5 \mathrm{~mL}$ das amostras e $2,5 \mathrm{~mL}$ da solução de DPPH na concentração de $40 \mu \mathrm{g} \mathrm{mL}^{-1}$ ) foram realizadas a $515 \mathrm{~nm}$ após um intervalo de 30 min de reação. Foi considerada como referência de máxima absorção, $0,5 \mathrm{~mL}$ da solução de DPPH adicionados a 2,5 mL de metanol. A partir da equação da curva de calibração e dos valores de absorvância no tempo de 30 min para cada concentração testada, foram determinados os percentuais de DPPH remanescentes (\% $\left.\mathrm{DPPH}_{\mathrm{Rem}}\right)$, conforme a Equação 1:

$$
\% \mathrm{DPPH}_{\mathrm{Rem}}=[\mathrm{DPPH}]_{\mathrm{T}=\mathrm{l}} /[\mathrm{DPPH}]_{\mathrm{T}=0} \times 100
$$

onde $[\mathrm{DPPH}]_{\mathrm{T}=\mathrm{t}}$ corresponde à concentração de DPPH no meio, após a reação com as amostras e $[\mathrm{DPPH}]_{\mathrm{T}=0}$ é a concentração inicial de DPPH, ou seja, $40 \mu \mathrm{g} \mathrm{mL} \mathrm{m}^{-1}$.

Os valores de DPPH no meio, após a reação com a amostra, foram calculados substituindo-se os valores das leituras obtidas para absorvância na equação da curva analítica. A partir dos valores de $\% \mathrm{DPPH}_{\text {Rem }}$ calculou-se a concentração eficiente, quantidade de antioxidante necessária para decrescer a concentração inicial de $\mathrm{DPPH}$ em $50 \%\left(\mathrm{CE}_{50}\right)$, graficando a porcentagem de $\mathrm{DPPH}_{\mathrm{Rem}}$ versus as concentrações de cada amostra. Os valores de absorvância em todas as concentrações testadas, no tempo de $30 \mathrm{~min}$, foram também convertidos em porcentagem de atividade antioxidante (AA), determinada pela Equação 2:

$$
\% \mathrm{AA}=\frac{\mathrm{A}_{0}-\mathrm{A}}{\mathrm{A}_{0}} \times 100
$$

onde $\mathrm{A}_{0}$ é absorvância do DPPH em metanol; A é a absorvância da mistura DPPH, metanol e amostra, ambos os casos após $30 \mathrm{~min}$ de reação; e \% AA é a atividade antioxidante em porcentagem. Com os percentuais das atividades antioxidantes das amostras foi construído um gráfico da \% AA versus a concentração de cada amostra.

\section{Estudos eletroquímicos}

O comportamento redox da quercetina e do complexo foi investigado por voltametria cíclica em potenciostato/galvanostato Metrohm Autolab PGSTAT 302 interfaceado a um microcomputador e gerenciado pelo software GPES (Versão 4.9) para aquisição dos dados. Os experimentos foram realizados em solução de acetonitrila sob atmosfera ambiente. Foi usado um sistema convencional de três eletrodos, composto por um eletrodo de carbono vítreo (diâmetro de 2,0 mm) como eletrodo trabalho, um eletrodo de $\mathrm{Ag} / \mathrm{Ag}^{+}$usado como eletrodo de referência e um fio de platina usado como eletrodo auxiliar (contraeletrodo). O eletrodo de trabalho foi cuidadosamente polido antes de cada experimento com alumina, lavado com água bidestilada e posteriormente seco. Para este sistema, foi usada uma célula convencional com capacidade máxima de $10,0 \mathrm{~mL}$ para as medidas. Uma solução de hexafluorofosfato de tetrabutilamônio $\left(0,1 \mathrm{mmol} \mathrm{L}^{-1}\right)$ foi utilizada como eletrólito suporte. Para monitorar o eletrodo de referência utilizou-se o par redox ferrocínio/ferroceno $\left(\mathrm{Fc}^{+} / \mathrm{Fc}\right.$ ) como padrão interno ${ }^{25}$ (onde $\mathrm{E}_{1 / 2(\mathrm{Fc}+/ \mathrm{Fc})}=0,950 \mathrm{~V}$ vs Ag/ $/ \mathrm{Ag}^{+}$ $\left.\operatorname{com} \Delta_{\text {Ep }}=0,120 \mathrm{~V}\right)$. 


\section{Síntese do complexo}

O complexo $\left[\mathrm{Ga}\left(\mathrm{C}_{15} \mathrm{H}_{9} \mathrm{O}_{7}\right)_{3}\right] \cdot 2 \mathrm{H}_{2} \mathrm{O} \cdot 2 \mathrm{CH}_{3} \mathrm{OH} \cdot \mathrm{CH}_{3} \mathrm{CH}_{2} \mathrm{OH}$ foi obtido reagindo-se o flavonoide quercetina com o sal nitrato de gálio(III), $\mathrm{Ga}\left(\mathrm{NO}_{3}\right)_{3} \cdot \mathrm{H}_{2} \mathrm{O}$ (Sigma-Aldrich), em uma estequiometria 3:1 (ligante:metal). A uma solução de $0,064 \mathrm{~g}$ de nitrato de gálio(III) em $10 \mathrm{~mL}$ de água, com agitação e aquecimento, adicionou-se uma solução da quercetina $(0,226 \mathrm{~g})$ solubilizada em aproximadamente 10 $\mathrm{mL}$ de etanol. A solução amarela continuou sob agitação e leve aquecimento por $20 \mathrm{~min}$, sendo adicionadas à mesma aproximadamente 3 gotas de uma solução tampão pH 4,5 (acetato de sódio/ácido acético), o que resultou na mudança de coloração para laranja. Manteve-se a reação em refluxo por $4 \mathrm{~h}$. Após alguns dias obteve-se a formação de um sólido amorfo de coloração verde, o qual foi filtrado, lavado com metanol a frio, seco e armazenado para a realização das análises.

\section{RESULTADOS E DISCUSSÃO}

\section{Análise elementar, espectroscopia (IV e UV-Vis) e estudo térmico}

O complexo foi obtido com grau de pureza adequado, o que é demonstrado pela análise elementar. A análise elementar apresenta resultado concordante com a fórmula molecular $\mathrm{GaC}_{49} \mathrm{H}_{45} \mathrm{O}_{26}(\mathrm{MM}$ $=1.119,60 \mathrm{~g} \mathrm{~mol}^{-1}$; calculado: $\mathrm{C}=52,57 \%$ e $\mathrm{H}=4,05 \%$; encontrado: $\mathrm{C}=52,25 \%$ e $\mathrm{H}=3,87 \%$ ), o que indica que o complexo mononuclear obtido foi isolado em uma estequiometria 3:1 (ligante/metal), contendo ainda duas moléculas de água, duas de metanol e uma de etanol como compostos de solvatação. Os resultados obtidos através da análise termogravimétrica reforçam esta sugestão.

Macedo e colaboradores ${ }^{26} \mathrm{em}$ um estudo sobre a caracterização térmica da quercetina mostraram que a mesma apresenta diferentes estágios de degradação, dependentes da atmosfera gasosa utilizada e velocidade de aquecimento, e que são relacionados com a perda de água (estágio inicial), transição de fase (estágio intermediário) e decomposição da quercetina (estágio final). O pico de transição de fase (curva DSC) ocorre sem a perda de massa (na curva TG), mas com variação de calor, o que evidencia uma mudança estrutural. Quando se compara o perfil das curvas de TG e DSC obtidas para o complexo (Figura 2) com o que é relatado para a quercetina, verifica-se uma similaridade nos estágios de decomposição, com uma diferença no início e final das curvas em virtude da presença de diferentes solventes e presença do íon metálico. A etapa de degradação inicial é caracterizada por processos endotérmicos relacionados com a evaporação de compostos orgânicos e água de solvatação, que se inicia em 26 ${ }^{\circ} \mathrm{C}$ e se estende até $130{ }^{\circ} \mathrm{C}$. Observam-se inflexões na curva dTG em 45 e $79^{\circ} \mathrm{C}$, indicando que no processo de evaporação há diferentes compostos envolvidos. A primeira perda de massa $(6,0 \%)$ está relacionada a duas moléculas de metanol, enquanto a subsequente $(7,6 \%)$ se relaciona com a mistura azeotrópica formada por duas moléculas de água e uma de etanol. Na etapa seguinte, similarmente ao que ocorre na quercetina livre, ${ }^{26}$ verifica-se um pico de transição de fase em $265^{\circ} \mathrm{C}$ (curva DSC), que ocorre sem perda de massa na curva TG, e que está atrelada a uma mudança estrutural, inalterada mesmo após o processo de coordenação. A etapa final, entre 325 e $525^{\circ} \mathrm{C}$, mostra o processo exotérmico associado à decomposição das moléculas de quercetina, com uma perda de massa total de $78,7 \%$. As três subetapas nesta decomposição estão associadas a diferentes estágios da combustão, como função da estabilidade térmica de distintas partes da molécula do ligante quercetina. A perda total de massa encontrada na TG foi de $92,3 \%$ e o total calculado foi de $93,4 \%$ com um resíduo final referente ao óxido de gálio. Estes resultados mostram boa concordância com a fórmula encontrada na análise elementar de $\mathrm{CHN}$ e permitiram estabelecer a estequiometria do complexo, o qual apresenta três moléculas de quercetina coordenadas ao centro metálico e moléculas de água, metanol e etanol como espécies de solvatação.
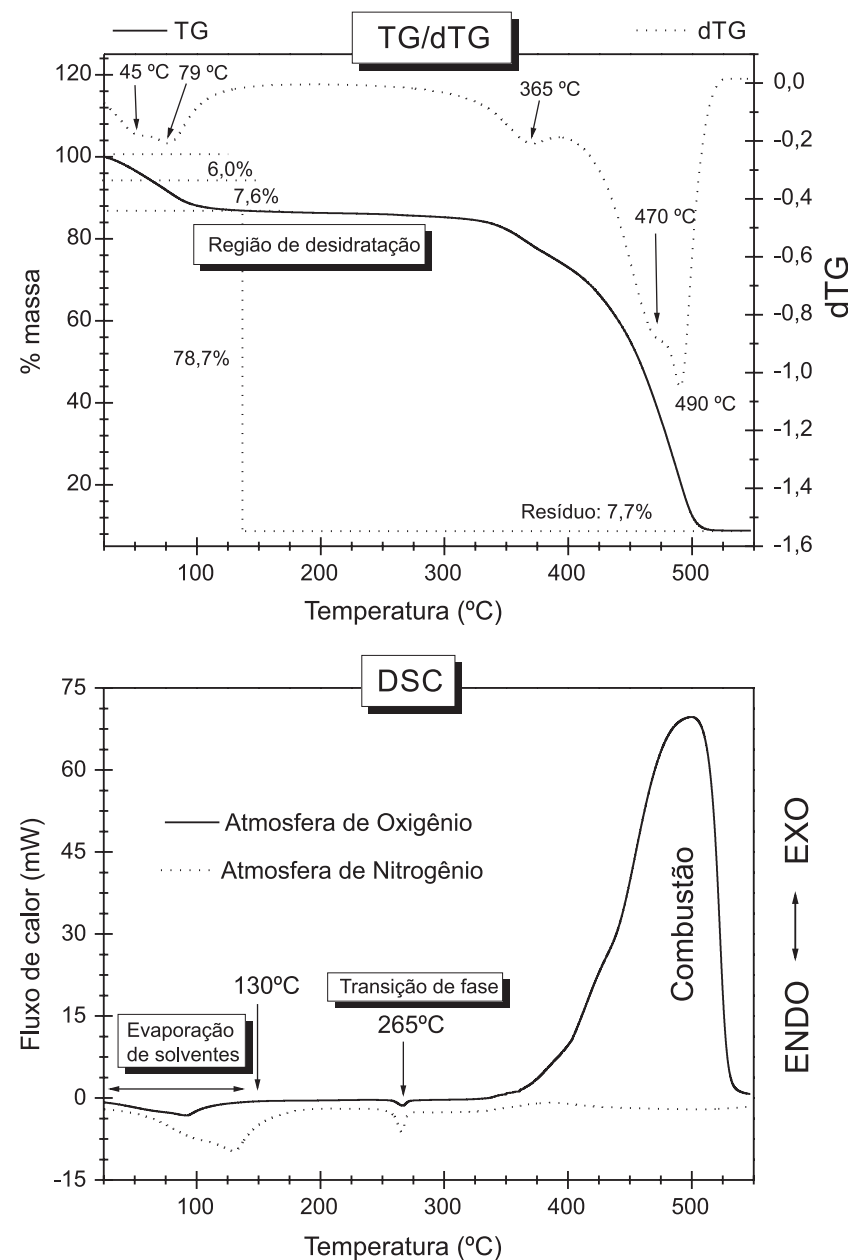

Figura 2. (Superior) Curva TG do complexo em atmosfera ambiente na faixa de temperatura $25-550^{\circ} \mathrm{C}$, coeficiente de aquecimento de $20^{\circ} \mathrm{C} \mathrm{min}^{-1} \mathrm{com}$ uma massa inicial da amostra de 7,5 mg. (Inferior) Curvas DSC do complexo sob atmosferas de nitrogênio e oxigênio (fluxo de $50,0 \mathrm{~mL} \mathrm{~min}{ }^{-1}$ ) com gradiente de aquecimento $20^{\circ} \mathrm{C} \mathrm{min}^{-1}$ e faixa de varredura entre 25 a $550^{\circ} \mathrm{C}$

Uma análise comparativa entre os espectros na região do infravermelho do ligante e do complexo indica a presença das principais bandas (Tabela 1), com algumas pequenas distinções oriundas do processo de coordenação. A presença da absorção em $448 \mathrm{~cm}^{-1}$ possivelmente está relacionada à formação das ligações $\mathrm{Ga}-\mathrm{O}$, pois relatos na literatura indicam que este tipo de ligação é encontrado em uma faixa de 450 a $470 \mathrm{~cm}^{-1}$, entretanto, com outros tipos de ligantes. ${ }^{10,27}$ Uma outra alteração é verificada na banda atribuída ao grupo carbonila que é deslocada para um menor comprimento de onda no complexo ( $1666 \mathrm{~cm}^{-1}$ na quercetina livre para $1650 \mathrm{~cm}^{-1}$ no complexo). Isto pode ser indicativo da coordenação do ligante ao íon metálico via oxigênio carbonílico (na posição 4 do anel C, Figura 1) uma vez que resultados similares foram encontrados para outros complexos com flavonolatos. ${ }^{2,3,5,28-35}$ Nota-se um aumento significativo da ordem de ligação da banda referente à deformação da ligação $\mathrm{C}-\mathrm{OH}_{\text {fenol }}$, de $1319 \mathrm{~cm}^{-1}$ no ligante para $1342 \mathrm{~cm}^{-1}$ no complexo, conforme pode ser verificado na Figura 3. Esse fato pode estar relacionado à coordenação de um dos grupamentos fenólicos presentes na quercetina (3-OH, 5-OH, 3'-OH e/ou 4'-OH). Como o grupo 3-hidroxil possui 
um próton mais ácido comparativamente aos demais (o que o torna um quelante mais efetivo), ${ }^{28,29}$ espera-se que seja um dos primeiros sítios, juntamente com o grupo 4-oxo, a serem envolvidos no processo de coordenação. Os demais grupos fenólicos não devem estar envolvidos devido à menor acidez e ao possível impedimento espacial ocasionado pela primeira complexação. ${ }^{2,29}$ Estudos com íons metálicos e flavonoides (principalmente a quercetina) têm indicado uma predominância nessas posições de coordenação, o que também é corroborado pelos dados obtidos através da espectroscopia eletrônica. ${ }^{2,3,5,28-35} \mathrm{~A}$ frequência de vibração para ${ }_{v}(\mathrm{C}-\mathrm{O}-\mathrm{C})$ também foi alterada após a coordenação do flavonoide, conforme pode ser visualizado na Figura 3. A partir desses resultados pode-se inferir que cada quercetina provavelmente se coordenou ao íon $\mathrm{Ga}(\mathrm{III})$ como um ligante bidentado através da carbonila (4-oxo) e do grupo 3-hidroxil. A presença das moléculas de metanol e etanol na estrutura do complexo promove uma ligeira modificação no perfil do espectro infravermelho, principalmente nas regiões esperadas para os modos vibracionais desses solventes. ${ }^{28} \mathrm{~A}$ presença dessas moléculas é devidamente confirmada pelas análises elementar e térmica.

Tabela 1. Principais bandas e atribuições, em $\mathrm{cm}^{-1}$, dos espectros no infravermelho para a quercetina e o complexo (detecção por fotoacústica com resolução $8 \mathrm{~cm}^{-1}$ e média de 256 espectros)

\begin{tabular}{ccc}
\hline Quercetina & Complexo & Atribuições \\
\hline 3394 & $\sim 3442-3285$ & ${ }_{\mathrm{v}}(\mathrm{O}-\mathrm{H})$ \\
1666 & 1650 & ${ }_{\mathrm{v}}(\mathrm{C}=\mathrm{O})$ \\
1250 & 1271 & ${ }_{\mathrm{v}}(\mathrm{C}-\mathrm{O}-\mathrm{C})$ \\
1319 & 1342 & ${ }_{\delta}\left(\mathrm{C}-\mathrm{OH}{ }_{\text {fenol }}\right)$ \\
-- & 448 & ${ }_{\mathrm{v}}(\mathrm{M}-\mathrm{O})$ \\
\hline
\end{tabular}

O espectro eletrônico da quercetina em metanol (Figura 4, inserção) apresenta duas bandas de absorção relacionadas com as transições $\pi \rightarrow \pi^{*}$ : uma em $375 \mathrm{~nm}\left(\varepsilon=24.167 \mathrm{~mol}^{-1} \mathrm{dm}^{3} \mathrm{~cm}^{-1}\right)$, denominada banda I, correspondente ao sistema conjugado entre o anel B e a carbonila do anel C (sistema cinamoil), e outra em $255 \mathrm{~nm}$ $\left(\varepsilon=24.580 \mathrm{~mol}^{-1} \mathrm{dm}^{3} \mathrm{~cm}^{-1}\right)$, banda II, referente ao sistema conjugado entre o anel A e a carbonila do anel C (sistema benzoil). ${ }^{2,28,29}$ Os espectros na região do UV-Vis em metanol mostram diferenças significativas entre a quercetina pura e o complexo (Figura 4), demonstrando claramente a coordenação do centro metálico: verificam-se o deslocamento batocrômico da banda I e o surgimento de uma nova

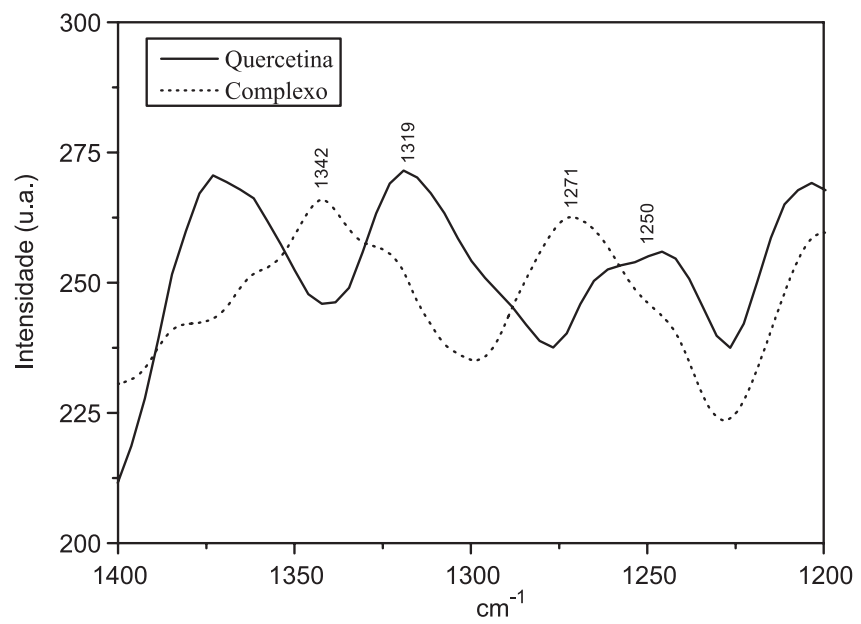

Figura 3. Espectro no infravermelho comparando a quercetina livre (linha sólida) e o complexo (linha tracejada) na região entre 1400 e $1200 \mathrm{~cm}^{-1}$ banda em $437 \mathrm{~nm}$ (com um ombro em $\approx 501 \mathrm{~nm}$ ). A nova banda em $437 \mathrm{~nm}\left(\varepsilon=30.500 \mathrm{~mol}^{-1} \mathrm{dm}^{3} \mathrm{~cm}^{-1}\right)$ sugere que o íon $\mathrm{Ga}$ (III) esteja ligado ao grupo fenólico 3-OH e à carbonila 4-oxo do anel C. ${ }^{36}$ Segundo Dehghan, ${ }^{28}$ as evidências que suportam esta sugestão estão relacionadas aos seguintes fatos: o grupo 3-OH é um melhor agente quelante (o que está relacionado com sua maior acidez frente aos demais) e a deslocalização dos elétrons do oxigênio do grupo 3-OH é maior, o que facilita a deslocalização dos elétrons $\pi$. O deslocamento batocrômico que ocorre na banda I (de $375 \mathrm{~nm}$ na quercetina para $388 \mathrm{~nm}$ no complexo) pode ser explicado pela interação do $\mathrm{Ga}^{3+}$ com o grupo 3-hidroxil da quercetina, resultando em uma redistribuição eletrônica entre a molécula do flavonoide e o íon metálico, formando um sistema ligante $\pi$ estendido. ${ }^{28}$ Baseado em relatos da literatura, sugere-se que o processo em $\approx 501 \mathrm{~nm}$ (ombro) pode estar relacionado ao fenômeno da fluorescência, que é explicado pela excitação e relaxamento dos elétrons do ligante $(\mathrm{n} \rightarrow \pi *$ ), influenciados pelo centro metálico. ${ }^{12}$ As mudanças após a coordenação são concordantes com o que é descrito em outros trabalhos que estudaram complexos metalo-flavonolatos, indicando, por exemplo, um deslocamento para menor comprimento de onda da banda referente ao sistema cinamoil (banda I). Como citado anteriormente, o grupo 3-hidroxil possui um próton mais ácido; dessa forma, os grupos 3-OH e 4-oxo são os primeiros sítios de coordenação envolvidos no processo de complexação e influenciam no deslocamento da banda. O grupo hidroxila localizado na posição 5 não é envolvido no processo de coordenação devido a sua menor acidez e ao impedimento espacial ocasionado pela primeira complexação. ${ }^{28,29}$

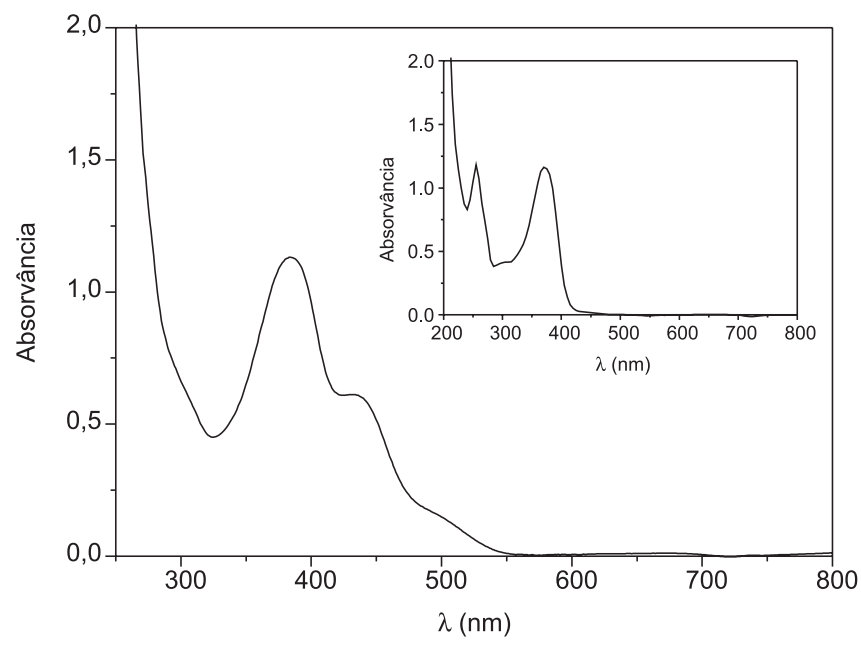

Figura 4. Espectro eletrônico do complexo em $\mathrm{CH}_{3} \mathrm{OH}$ (concentração: 2, $0 x$ $10^{-5} \mathrm{~mol} \mathrm{~L}^{-1}$; caminho óptico: $1 \mathrm{~cm}$ ). Inserção: espectro eletrônico da quercetina em metanol (concentração: 4,8 $\times 10^{-5} \mathrm{~mol} \mathrm{~L}^{-1}$; caminho óptico: $1 \mathrm{~cm}$ )

\section{Ensaio citotóxico}

Vários estudos correlacionam a toxicidade sobre a A. salina com atividades antifúngica, antimicrobiana, tripanossomicida e parasiticida e, também, como avaliação prévia de uma possível atividade antitumoral. O microcrustáceo pode ser utilizado para estimar a toxicidade através da concentração média letal $\left(\mathrm{DL}_{50}\right)$, que é a dose efetiva para matar $50 \%$ das larvas de A. salina. ${ }^{15-17}$

Os resultados obtidos no ensaio com A. salina (Tabela 2) indicam que todas as amostras se mostraram ativas contra o microcrustáceo, pois apresentaram resultados de $\mathrm{DL}_{50}$ inferiores a $1000 \mu \mathrm{g} \mathrm{mL}^{-1}$, que é a concentração máxima para uma amostra ser considerada ativa. ${ }^{15} \mathrm{O}$ pré-controle feito com metanol e água salina não apresentou influência sobre os resultados, já que nenhuma larva morreu nessa mistura. $\mathrm{O}$ 
valor de $\mathrm{DL}_{50}$ para o complexo $\left(\mathrm{DL}_{50}=22,51 \mu \mathrm{g} \mathrm{mL}^{-1}\right)$ indica uma melhor resposta citotóxica do composto, sendo consideravelmente menor que o da quercetina pura $\left(\mathrm{DL}_{50}=398,94 \mu \mathrm{g} \mathrm{mL}^{-1}\right)$ e o do lapachol $\left(\mathrm{DL}_{50}=64,85 \mu \mathrm{g} \mathrm{mL}^{-1}\right)$. O valor de $\mathrm{DL}_{50}$ obtido para o lapachol é um valor muito próximo ao encontrado na literatura $\left(68 \mu \mathrm{g} \mathrm{mL}^{-1}\right),{ }^{37}$ o que indica boa adequação nos procedimentos experimentais utilizados.

Tabela 2. Resultados do teste de citotoxicidade sobre A. salina, relativos à quercetina, ao complexo e à substância controle (lapachol)

\begin{tabular}{ccc}
\hline Amostras & $\left.\mathrm{DL}_{50}(\mu \mathrm{g} \mathrm{mL})^{-1}\right)^{*}$ & Intervalo de confiança (95\%) \\
\hline Complexo & $22,51 \pm 0,11$ & $7,57-40,97$ \\
Quercetina & $398,94 \pm 0,04$ & $339,42-496,86$ \\
Lapachol & $64,85 \pm 0,05$ & $48,56-98,52$ \\
\hline
\end{tabular}

* Dados apresentados como médias \pm desvio padrão de 3 repetições.

\section{Avaliação da atividade antioxidante}

O modelo para avaliação da atividade antioxidante utilizando DPPH é baseado na capacidade do radical livre estável 2,2-difenil-1-picril-hidrazil em reagir com substâncias doadoras de hidrogênio, incluindo compostos fenólicos..$^{24,38}$ Quando uma determinada substância que age como doadora de átomos de hidrogênio é adicionada a uma solução de DPPH, a hidrazina (forma reduzida) é obtida com mudança simultânea na coloração de violeta para amarela pálida. É um método fácil, rápido e muito utilizado para avaliar a capacidade sequestradora de radicais livres de amostras.$^{39}$ Os resultados da avaliação da atividade antioxidante (\%AA) do complexo e do controle positivo, determinados pelo ensaio do DPPH, estão apresentados na Figura 5, mostrando que o complexo apresentou boa atividade antioxidante, chegando a ser mais efetivo que a quercetina pura em todas as concentrações testadas.

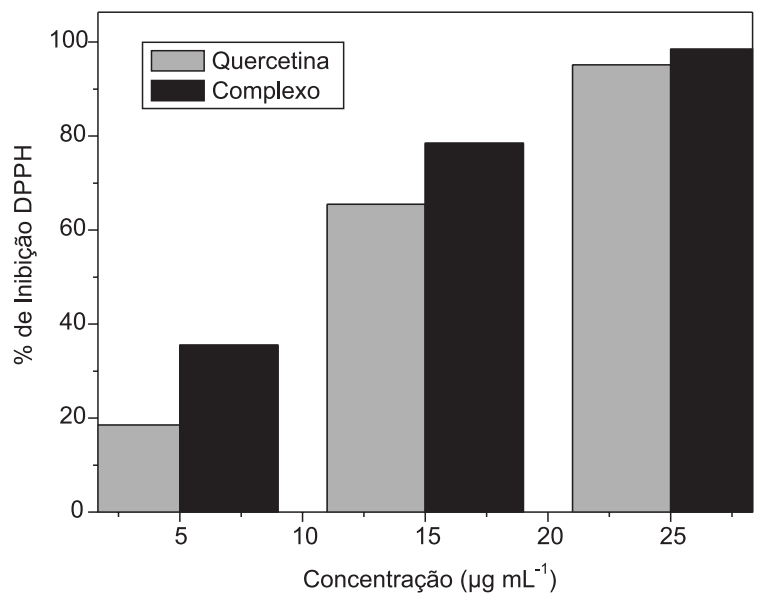

Figura 5. Porcentagem da atividade de inibição do DPPH da quercetina e do complexo

A concentração de cada amostra necessária para decrescer a concentração inicial de DPPH em 50\%, $\mathrm{CE}_{50}$, também indicou a potencialização da atividade antioxidante do flavonoide quercetina após a complexação com o íon $\mathrm{Ga}^{3+}$. O complexo apresentou um valor de $\mathrm{CE}_{50}$ de $0,73 \mu \mathrm{g} \mathrm{mL}^{-1}$. Comparativamente, a quercetina apresentou um valor de $2,4 \mu \mathrm{g} \mathrm{mL} \mathrm{m}^{-1}$, similar aos encontrados na literatura, ${ }^{24,40}$ que variam de 2,20 a 2,34 $\mu \mathrm{g} \mathrm{mL}^{-1}$. A capacidade dos flavonoides de atuarem como antioxidantes depende da sua estrutura molecular; entretanto, há muitas divergências quanto ao mecanismo envolvido no processo. ${ }^{41-45}$ Alguns autores sugerem que a atividade está relacionada com a posição e o número de grupamentos hidroxílicos presentes na estrutura do flavonoide. A alta atividade antioxidante poderia ser atribuída, principalmente, à contribuição significativa dos grupos 3',4'-OH. A quercetina em reação com o radical DPPH doaria um dos átomos de hidrogênio e se transformaria em uma espécie radicalar (Q`) ou uma semiquinona, sendo esta reação atribuída à presença das hidroxilas em posição orto no anel B. Entretanto, recentemente, Foti e colaboradores ${ }^{45}$ sugeriram que a etapa inicial do processo deve ser representada pela transferência de elétrons entre o DPPH e o oxigênio fenólico na posição C-7, em vez da abstração de um dos átomos de hidrogênio dos grupos 3',4'-OH.

$\mathrm{O}$ aumento da atividade antioxidante da quercetina após a complexação sugere que o íon metálico $\mathrm{Ga}^{3+}$ pode alterar as propriedades químicas do ligante quercetina, retirando densidade eletrônica dos grupos fenólicos circunvizinhos, fazendo com que se tornem mais ácidos e, consequentemente, mais susceptíveis de serem abstraídos ou permitirem a transferência eletrônica. Dados experimentais, utilizando diversos íons metálicos, têm demonstrado que os complexos são consideravelmente mais eficientes sequestradores de radicais livres do que os flavonoides livres. , 7,46 Os resultados obtidos abrem uma nova perspectiva para o uso do complexo de gálio como fármaco, visando a prevenção de doenças decorrentes do estresse oxidativo. No entanto, o teste utilizando o DPPH não permite uma definição dos efeitos antioxidantes, sendo necessário um estudo utilizando um sistema biológico, como células, para determinar se este composto poderá ser utilizado para esta finalidade.

\section{Estudos eletroquímicos}

O comportamento eletroquímico da quercetina e do complexo foi investigado através da técnica de voltametria cíclica. Os voltamogramas obtidos são apresentados na Figura 6. Os processos foram obtidos em solução de acetonitrila, utilizando carbono vítreo como eletrodo de trabalho, $\mathrm{Ag} / \mathrm{Ag}^{+}$como eletrodo de referência, fio de platina como eletrodo auxiliar e $0,1 \mathrm{mmol} \mathrm{L}^{-1}$ de $\mathrm{TBAPF}_{6}$ como eletrólito suporte. Todos os valores dos potenciais foram referenciados versus $\mathrm{Fc}^{+} / \mathrm{Fc},{ }^{25}$ onde $\mathrm{E}_{1 / 2}\left(\mathrm{Fc}^{+} / \mathrm{Fc}\right)=0,950 \mathrm{~V}$ vs $\mathrm{Ag} / \mathrm{Ag}^{+}$ $\left(\Delta_{\mathrm{Ep}}=0,120 \mathrm{~V}\right)$ (Figura 6, inserção). No voltamograma cíclico obtido para a quercetina em solução de acetonitrila $\left(0,8 \mathrm{mmol} \mathrm{L}^{-1}\right)$

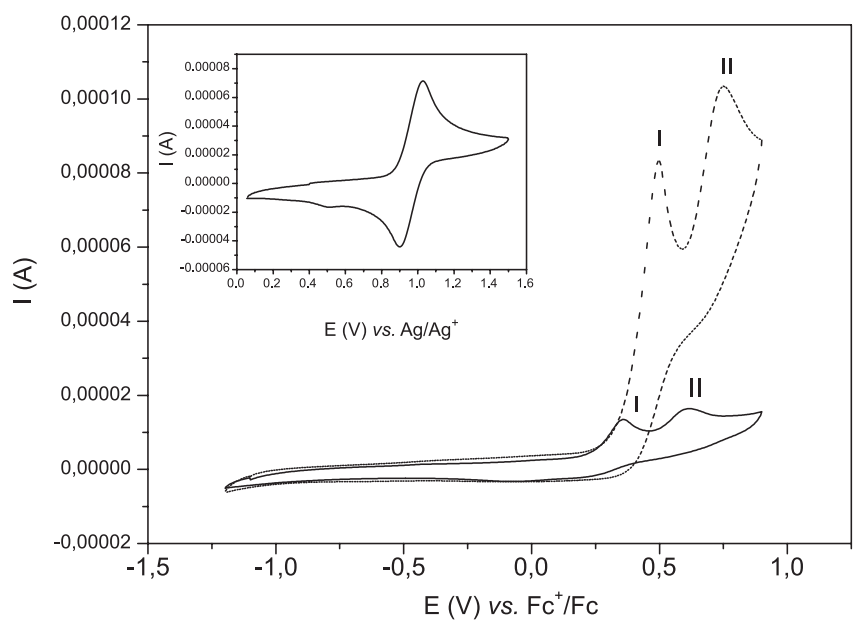

Figura 6. Voltamogramas cíclicos da quercetina (linha tracejada) e do complexo (linha sólida). Inserção: voltamograma do par redox ferrocínio/ ferroceno $\left(\mathrm{Fc}^{+} / \mathrm{Fc}\right)$ utilizado como padrão interno $\left(E_{1 / 2}=0,95 \mathrm{Vvs}\right.$. $\left.\mathrm{Ag}_{\mathrm{Ag}}\right)$. Condições experimentais similares para todos os voltamogramas (solvente: acetonitrila; eletrodo trabalho: carbono vítreo; eletrodo de referência: $\mathrm{Ag} /$ $\mathrm{Ag}^{+}$; eletrodo auxiliar: fio de platina; eletrólito suporte: 0,1 $\mathrm{mmol} \mathrm{L}^{-1} \mathrm{TBAPF}_{6}$; velocidade de varredura de $25 \mathrm{mV} \mathrm{s}^{-1}$ ) 
para uma velocidade de varredura de $25 \mathrm{mV} \mathrm{s}^{-1}$ são observados dois processos de oxidação irreversíveis em $0,553 \mathrm{~V}$ vs $\mathrm{Fc}^{+} / \mathrm{Fc}\left(\mathrm{E}_{\mathrm{pal}}\right)$ e $0,807 \mathrm{~V}$ vs $\mathrm{Fc}^{+} / \mathrm{Fc}\left(\mathrm{E}_{\mathrm{paII}}\right)$. De acordo com dados já reportados na literatura, ${ }^{47-50} \mathrm{o}$ processo no pico $\mathrm{I}\left(\mathrm{E}_{\mathrm{paI}}\right)$ corresponde à oxidação do grupo 3',4'-di-hidroxil no anel B da quercetina, em uma reação envolvendo os prótons. O grupo hidroxila na posição 5- do anel A é oxidado depois e corresponde ao pico II $\left(\mathrm{E}_{\mathrm{paII}}\right)$, enquanto a reação de oxidação envolvendo o grupo 7-hidroxil não é observada no voltamograma cíclico nas condições utilizadas. Quanto à reversibilidade das reações que ocorrem nesses processos de oxidação, ainda é contraditória e depende muitas vezes dos parâmetros experimentais empregados. . $^{47,48,50}$

No voltamograma cíclico do complexo, seguindo as mesmas condições experimentais utilizadas para a quercetina pura, verificam-se os mesmos processos descritos anteriormente, entretanto, com diferenças nos valores dos potenciais. Esses resultados mostraram uma diminuição considerável dos potenciais de oxidação (pico I: $0,305 \mathrm{~V}$ vs $\mathrm{Fc}^{+} / \mathrm{Fc}$ e pico II: $0,565 \mathrm{~V}$ vs $\mathrm{Fc}^{+} / \mathrm{Fc}$ ) comparados ao ligante livre, pois a coordenação com o íon metálico faz com que os processos de oxidação ocorram mais facilmente. ${ }^{2}$ Não há nenhum processo relacionado diretamente ao íon metálico $\mathrm{Ga}(\mathrm{III})$, uma vez que o mesmo é inerte eletroquimicamente. ${ }^{51}$

Através de técnicas eletroanalíticas, como a voltametria cíclica, é possível determinar a atividade antioxidante de substâncias. Essas técnicas podem correlacionar potenciais de oxidação, intensidade de corrente e/ou outros parâmetros eletroquímicos com a capacidade antioxidante, sendo mais seletivas e sensíveis que os métodos espectrofotométricos. Quanto menor o valor de $\mathrm{E}_{\mathrm{pa}}$, maior é o poder doador de elétron da espécie em estudo e, portanto, teoricamente, maior seu poder antioxidante. ${ }^{39}$ Assim, o complexo sintetizado mostrou-se um antioxidante mais eficaz do que a quercetina livre. Estes resultados concordam com o teste antioxidante utilizando o radical livre estável DPPH.

\section{CONSIDERAÇÕES FINAIS}

Em virtude do crescente interesse na química de complexos metal-flavonolatos, o presente trabalho estudou a interação entre a quercetina e o íon gálio(III), sendo que as análises físico-químicas realizadas sugerem a coordenação do $\mathrm{Ga}^{3+}$ através do grupo carbonila (4-oxo) e do oxigênio fenólico (na posição 3 do anel C) do flavonoide, formando um complexo mononuclear com fórmula $\left[\mathrm{Ga}\left(\mathrm{C}_{15} \mathrm{H}_{9} \mathrm{O}_{7}\right)_{3}\right] .2 \mathrm{H}_{2} \mathrm{O} .2 \mathrm{CH}_{3} \mathrm{OH} . \mathrm{CH}_{3} \mathrm{CH}_{2} \mathrm{OH}$. O complexo apresentou resposta citotóxica frente ao crustáceo $A$. salina mais efetiva quando comparado ao flavonoide puro e ao lapachol (utilizado como controle). No estudo da atividade antioxidante baseado no radical livre DPPH, observou-se um aumento nessa propriedade quando se comparou o complexo ao flavonoide livre, o que demonstra claramente a importância da coordenação do íon metálico para o processo. A atividade antioxidante do complexo também foi avaliada do ponto de vista eletroquímico, confirmando o resultado obtido com o teste utilizando o radical DPPH. Os resultados mostraram valores relativamente mais baixos nos potenciais de oxidação do complexo comparativamente aos da quercetina pura, o que indica que pode se oxidar com mais facilidade que o flavonoide e, consequentemente, ser melhor agente antioxidante. Todos estes resultados abrem espaço para a busca de outras atividades do complexo sintetizado, bem como uma melhor investigação das propriedades antioxidante e citotóxica, visando uma possível aplicação farmacêutica.

\section{AGRADECIMENTOS}

Às agências CAPES e FUNDECT.

\section{REFERÊNCIAS}

1. Simões, C. M. O.; Schenkel, E. P.; Gosmann, G.; Mello, J. C. P.; Mentz, L. A.; Petrovick, P. R.; Farmacognosia - da Planta ao Medicamento, $5^{\text {a }}$ ed., Ed. da UFSC: Florianópolis, 2004, cap. 23.

2. Souza, R. F. V.; Giovani, W. F.; Spectrochim. Acta, Part A 2005, 61, 1985.

3. Dolatabadi, J. E. N.; Int. J. Biol. Macromol. 2011, 48, 227.

4. Franzoi, A. C.; Peralta, R. A.; Neves, A.; Vieira, I. C.; Talanta 2009, 78, 221.

5. Berreau, L. M.; Grubel, K.; Rudzka, K.; Arif, A. M.; Klotz, K. L.; Halfen, J. A.; Inorg. Chem. 2010, 49, 82.

6. Behling, E. B.; Sendão, M. C.; Francescato, H. D. C.; Antunes, L. M. G.; Bianchi, M. L. P.; Alim. Nutr. 2004, 15, 285.

7. Zhay, G.; Ma, H.; Duan, Y.; Zhu, W.; Zir. Kex. 2010, 23, 310.

8. Bernstein, L. R.; Pharmacol. Rev. 1998, 50, 669.

9. Melnikov, P.; Malzac, A.; Coelho, M. B.; Acta Ortop. Bras. 2008, 16, 54.

10. Bandoli, G.; Dolmella, A.; Tisato, F.; Porchia, M.; Refosco, F.; Coord. Chem. Rev. 2009, 253, 56.

11. Silva, F. A. A. C.; Dissertação de Mestrado, Universidade de Lisboa, Portugal, 2008.

12. Kopacz, M.; Bartecki, A.; Koord. Khim. 1978, 4, 1845.

13. Kopacz, M.; Bartecki, A.; Koord. Khim. 1979, 5, 367.

14. Bujonek, B.; Kopacz, M.; Pol. J. Chem. 1991, 65, 845.

15. Meyer, N. B.; Ferrigni, N. R.; Jacobson, J. E.; Nichols, D. E.; McLaughein, J. L.; Plan. Méd. 1982, 45, 31.

16. Siqueira, J. M.; Bomm, M. D.; Pereira, N. F. G.; Garcez, W. S.; Boaventura, M. A.; Quim. Nova 1998, 21, 557.

17. Citó, A. M. G. L.; Souza, A. A.; Lopes, J. A. D.; Chaves, M. H.; Costa, F. B.; Souza, S. A. A.; Amaral, M. P. M.; An. Assoc. Bras. Quím. 2003, 52,74 .

18. Ferreira, M. L.; Barbosa, L. C. A.; Demuner, A. J.; da Silva, A. A.; Pereira, R. C.; Acta Scient. 2000, 22, 999.

19. Hussain, H.; Krohn, K.; Ahmad, V. U.; Miana, G. A.; Green, I. R.; Arkivoc 2007, part ii, 145.

20. Maeda, M.; Murakami, M.; Takegami, T.; Ota, T.; Toxicol. Appl. Pharmacol. 2008, 229, 232.

21. Finney, D. J.; Probit Analysis, $3^{\text {rd }}$ ed.; Cambridge Univ. Press: Cambridge, 1971.

22. BioStat ${ }^{\oplus}$ for Windows, Versão 5.8.3.0, AnalystSoft Inc., Vancouver, 2009.

23. Sousa, C. M. M.; Silva, H. R.; Vieira Jr, G. M.; Ayres, M. C. C.; Costa, C. L. S.; Araujo, D. S.; Cavalcante, L. C. D.; Barros, E. D. S.; Araújo, P. B. M.; Brandão, M. S.; Chaves, M. H.; Quim. Nova 2007, 30, 351.

24. Barbosa, J.; Dissertação de Mestrado, Universidade Federal de Minas Gerais, Brasil, 2009.

25. Gagné, R. R.; Koval, C. A.; Lisensky, G. C.; Inorg. Chem. 1980, 19, 2854.

26. Costa, E. M.; Barbosa Filho, J. M.; Nascimento, T. G.; Macedo, R. O.; Thermochim. Acta 2002, 392-393, 79.

27. Mohammadi, K.; Thompson, K. H.; Patrick, B. O.; Storr, T.; Martins, C.; Polishchuk, E.; Yuen, V. G.; Mcneill, J. H.; Orvig, C.; J. Inorg. Biochem. 2005, 99, 2217.

28. Dehghan, G.; Khoshkam, Z.; Food Chem. 2012, 131, 422.

29. Bukhari, S. B.; Memon, S.; Tahir, M. M.; Bhanger, M. I.; Spectrochim. Acta, Part A 2009, 71, 1901.

30. Tian, Q-L.; Liao, S-H.; Lu, P.; Liu, L-J.; Chin. J. Chem. 2006, 24, 1388.

31. Woznicka, E.; Kopacz, M.; Umbreit, M.; Klos, J.; J. Inorg. Biochem. 2007, 101, 774

32. Li, J.; Kang, J.; Lu, J.; Li, X. Z.; Tang, J. L.; Zhang, H. Q.; Zhang, Y. H.; J. Lumin. 2009, 129, 906.

33. Kopacz, M.; Nowak, D.; Microchem. J. 1993, 47, 338. 
34. Kopacz, M.; Nowak, D.; Polish. J. Chem. 2000, 74, 303.

35. Zhou, J.; Wang, J.; Tang, N.; J. Inorg. Biochem. 2001, 83, 41.

36. Ahmadi, S. M.; Dehghan, G.; Hosseinpourfeizi, M. A.; Ezzati, N. D. J.; Kashanian, S.; DNA Cell. Biol. 2011, 30, 517.

37. Galotta, A. L. Q. A.; Boaventura, M. A. D.; Quim. Nova 2005, 28, 610.

38. Roginsky, V.; Lissi, E.; Food Chem. 2005, 92, 235.

39. Alves, C. Q.; David, J. M.; David, J. P.; Bahia, M. V.; Aguiar, R. M.; Quim. Nova 2010, 33, 2202.

40. Chua, M.; Tung, Y.; Chang, S.; Bioresour. Technol. 2008, 99, 1918.

41. Birt, D. F.; Hendrich, S.; Wang, W.; Pharmacol. Ther. 2001, 90, 157.

42. Yang, C. S.; Landau, J. M.; Huang, M. T.; Newmark, H. L.; Annu. Rev. Nutr. 2001, 21, 381.

43. Materska, M.; Pol. J. Food Nut. Sc. 2008, 58, 407.

44. Wang, L.; Tu, Y. C.; Lian, T. W.; Hung, J. T.; Yen, J. H.; Wu, M. J.; J. Agric. Food Chem. 2006, 54, 9798.
45. Foti, M. C.; Daquino, C.; Dilabio, G. A.; Ingold, K. U.; Org. Lett. 2011, $13,4826$.

46. Malešev. D.; Kuntic, V.; J. Serb. Chem. Soc. 2007, 72, 921.

47. Brett, A. M.; Ghica, M. E.; Electroanalysis 2003, 15, 1745.

48. Medvidović-Kosanović, M.; Šeruga, M.; Jakobek, L.; Novak, I.; Croat. Chem. Acta 2010, 83, 197.

49. Xu, G. R.; In, M. Y.; Yuan, Y.; Lee, J. J.; Kim, S.; Bull. Korean Chem. Soc. 2007, 28, 889 .

50. Timbola, A. K.; de Souza, C. D.; Giacomelli, C.; Spinelli, A.; J. Braz. Chem. Soc. 2006, 17, 139.

51. dos Anjos, A.; Bortoluzzi, A. J.; Caro, M. S. B.; Peralta, R. A.; Friedermann, G. R.; Mangrich, A. S.; Neves, A.; J. Braz. Chem. Soc. 2006, 17, 1540 . 